J3eA - Vol. 1 - 3 (2002).

DOI : 10.1051/bib-j3ea:2002003

\title{
Quelques exercices types sur le GRAFCET
}

\author{
D. Chappe * \\ Mis en ligne le 22 février 2002.
}

\begin{abstract}
Résumé
Cet article présente une collection d'exemples que nous utilisons pour l'enseignement du GRAFCET à l'école d'ingénieurs de Besançon (ENSMM). Ils correspondent aux principaux cas que l'on peut rencontrer (exercices types, exercices génériques). Les séances de travaux dirigés se font sur calculateur, les étudiants peuvent tester leur solution à l'aide d'une simulation. La plupart des exemples proposés ici sont illustrés par une animation obtenue par une copie d'écran lors de la simulation.
\end{abstract}

Mots-clés : GRAFCET, EAO, simulation.

(C) EDP Sciences, 2002.

Niveau de connaissances requis. Aucun prérequis n'est nécessaire.

Niveau des étudiants. Le cours est utilisé par les étudiants de première année de l'ENSMM (école d'ingénieurs) mais pourrait également utilisé en premier cycle.

* Daniel Chappe est né le 4 décembre 1945. Ingénieur ENSMM en 1969, docteur-ingénieur en 1973, il est assistant à l'ENSMM de 1969 à 1976, puis maître de conférences à l'ENSET Tunis (Tunisie) de 1976 à 1985. Depuis 1985 il est maître de conférences à l'ENSMM (école d'ingénieurs).

Daniel Chappe enseigne l'automatique (systèmes logiques, systèmes asservis) en première année à l'ENSMM et la modélisation et la simulation des systèmes de production en troisième année. Ses thèmes de recherches sont la productique et la modélisation de systèmes de production. Il est également responsable du centre de formation continue de l'ENSMM.

Adresse postale : Daniel Chappe, ENSMM, 26 chemin de l'Épitaphe, 25030 Besançon Cedex, France. email : dchappe@ens2m.fr 


\section{Introduction}

Les moyens multimédia peuvent apporter beaucoup à l'enseignement des disciplines scientifiques et techniques. À l'école d'ingénieurs de Besançon nous avons mis en place en 1998 un cours sur le GRAFCET utilisant ces moyens. Le cours magistral a été supprimé et remplacé par un cours au format html sur ordinateur [1]. Les étudiants ont ce cours à disposition lors des séances de travaux dirigés. Les étudiants consultent le cours puis font les exercices associés. Pour ces exercices des logiciels de simulation (Automgen et Iris de la société IRAI $[2,3,4])$ permettent de valider les solutions.

Le but de cet article n'est pas de présenter ce cours (qui est disponible sur le serveur du Laboratoire d'Automatique de Besançon), mais de présenter quelques exercices sur le GRAFCET qui illustrent ce cours et surtout leurs solutions sous forme d'images animées. Ces images ont été obtenues à partir de copies d'écrans lors de la simulation.

\section{Exercices types}

Ces exercices sont classiques et ont déjà été publiés [5,6], leur but est d'illustrer les configurations les plus couramment rencontrées.

\subsection{Tri de caisse (séquences au choix)}

Un dispositif automatique destiné à trier des caisses de deux tailles différentes se compose d'un tapis amenant les caisses, de trois poussoirs et de deux tapis d'évacuation (Fig. 1).

Le poussoir P1 pousse les petites caisses devant le poussoir P2 qui à son tour les transfère sur le tapis 2, alors que les grandes caisses sont poussées devant le poussoir P3, ce dernier les évacuant sur le tapis 3. Pour effectuer la sélection des caisses, un dispositif de détection placé devant le poussoir P1 permet de reconnaitre sans ambiguïté le type de caisse qui se présente ( $a=1$ si petite caisse, $b=1$ si grande caisse). La simulation utilise le modèle de la figure 2. Vous pouvez voir une animation de la solution au format AVI (40 Ko) ou au format GIF animé (14 Ko)

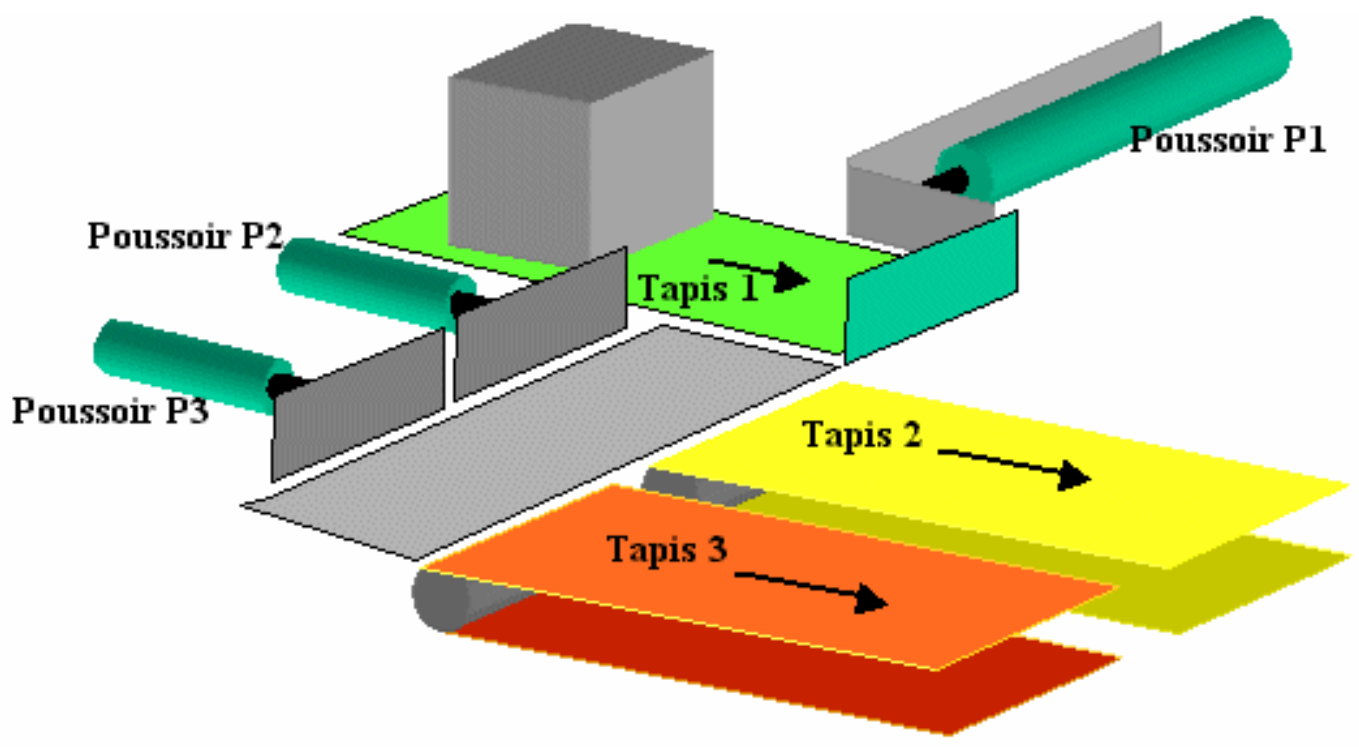

Figure 1. Dispositif de tri. 


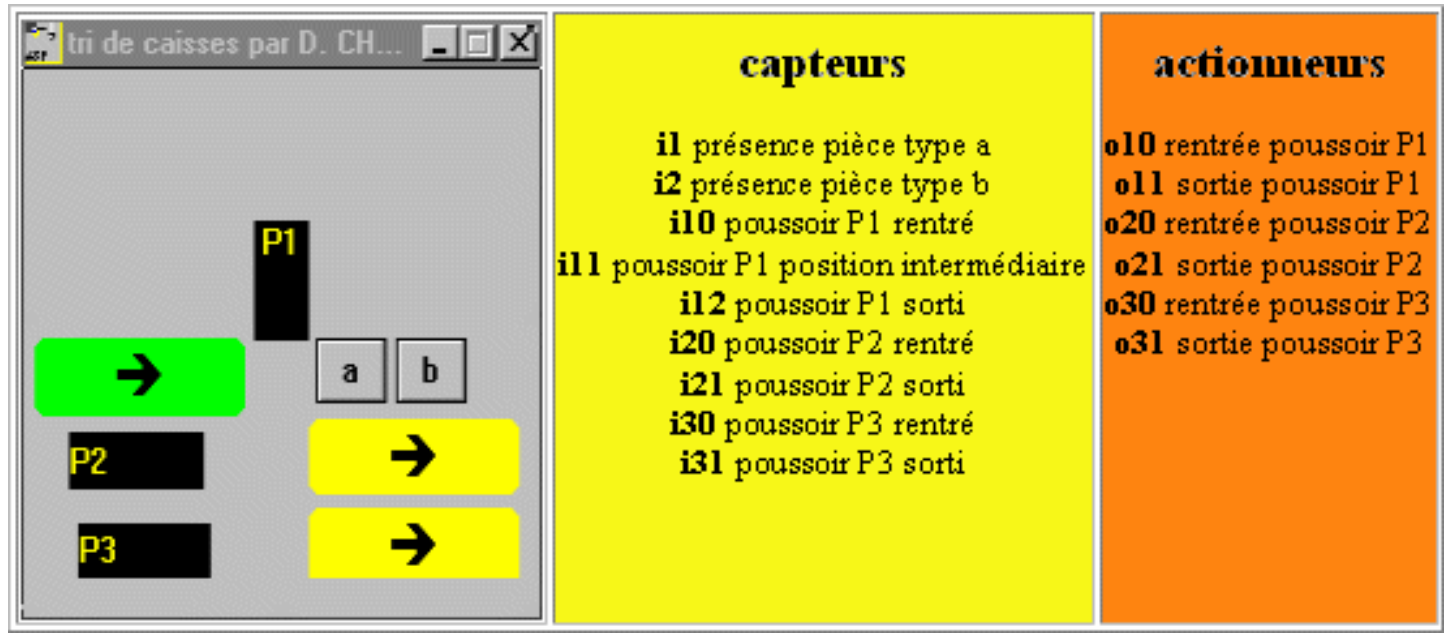

Figure 2. Modèle de simulation de la partie opérative.

\subsection{Poste de perçage (séquences parallèles)}

Un plateau tournant (Fig. 3) dessert trois postes de travail :

- un premier poste de chargement ;

- un deuxième de perçage ;

- un troisième de contrôle et d'évacuation des pièces percées.

Un vérin permet la rotation de $90^{\circ}$ du plateau supportant les pièces à usiner et son indexation, c'est-à-dire son blocage précis après chaque rotation. Le contrôle du perçage s'effectue par un testeur qui doit descendre en position basse, si le trou est correctement percé. Si cela n'est pas réalisé, tout le système se bloque, testeur en position haute, de façon à ce que l'opérateur puisse enlever la pièce défectueuse avant de réarmer manuellement le système. La simulation utilise le modèle de la figure 4 . Vous pouvez voir une animation de la solution au format AVI (109 Ko) ou au format GIF animé (24 Ko) 


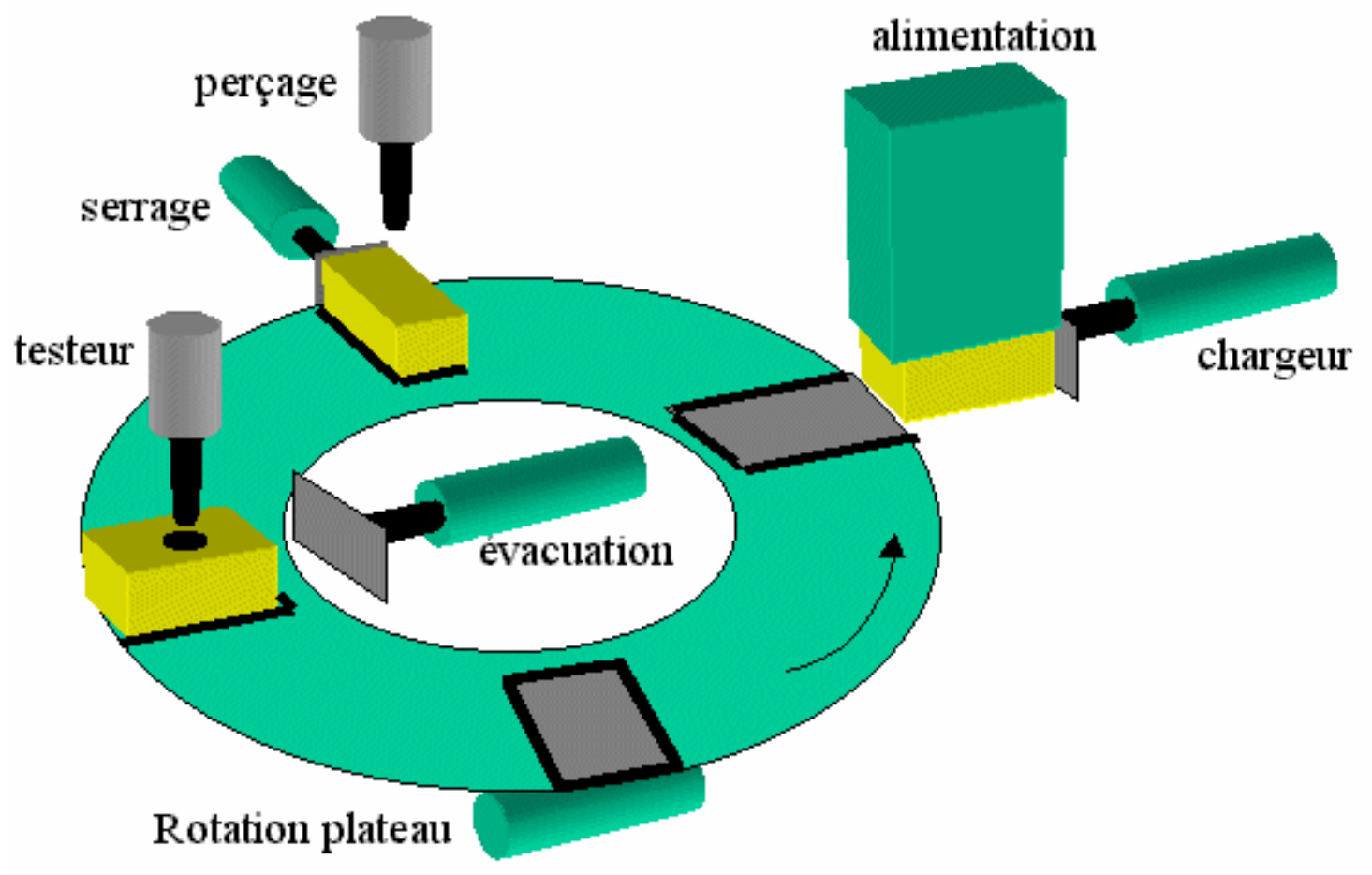

Figure 3. Poste de perçage.

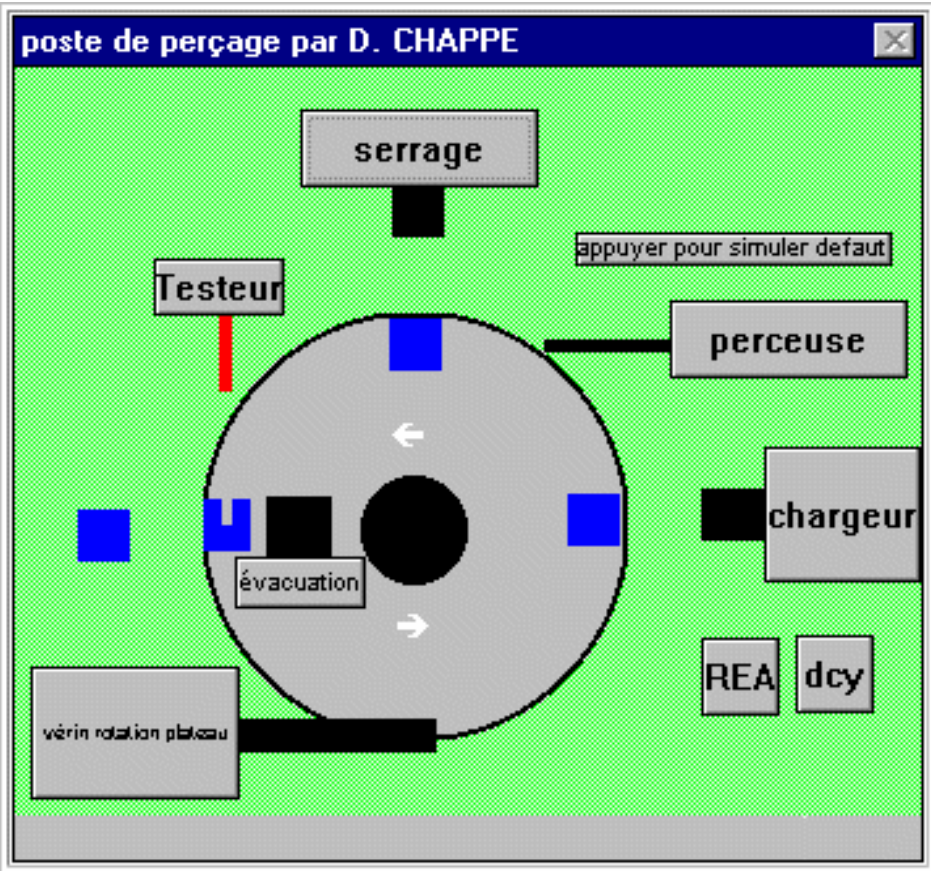

\section{actionneurs}

CH+ :avance chargeur

CH- :recul chargeur

SR+ :avance vérin serrage

SR- :recul vérin serrage

MONTEE : montée perceuse

DES CENTE :descente

perceuse

TE+ :descente testeur

TE- :montée testeur

EV+ :avance vérin évacuation

EV- :recul vérin évacuation

$\mathbf{0 3}$ :avance vérin rotation

plateau

04 :recul vérin rotation plateau

Figure 4. Modèle de simulation de la partie opérative.

\subsection{Commande de pompes (regroupement de séquences)}

Le niveau de liquide contenu dans un réservoir (Fig. 5) est contrôlé par trois détecteurs N1, N2, N3.

L'alimentation de ce réservoir s'effectue par trois pompes P1, P2, P3 de la façon suivante : 
- $\quad$ si le niveau $\mathrm{N} 1$ est découvert $(\mathrm{N} 1=0)$, une première pompe est mise en marche ;

- si le niveau $\mathrm{N} 2$ est découvert $(\mathrm{N} 1=0, \mathrm{~N} 2=0)$, une deuxième pompe est mise en marche ;

- si le niveau $\mathrm{N} 3$ est découvert $(\mathrm{N} 1=0, \mathrm{~N} 2=0, \mathrm{~N} 3=0)$, la troisième pompe est mise en marche.

Le nombre de pompes en service sera égal au nombre de niveaux découverts. De plus afin d'équilibrer l'usure des pompes, celles-ci seront permutées à tour de rôle. La simulation utilise le modèle de la figure 6. Vous pouvez voir une animation de la solution au format AVI (58 Ko) ou au format GIF animé (23 Ko)

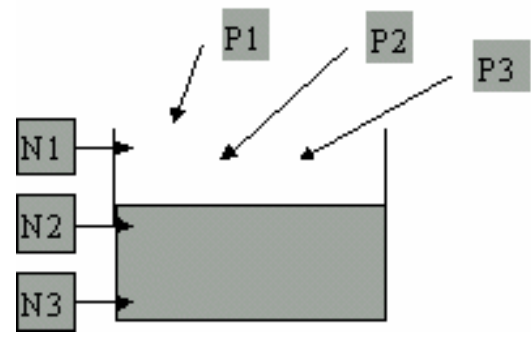

Figure 5. Commande de pompes.

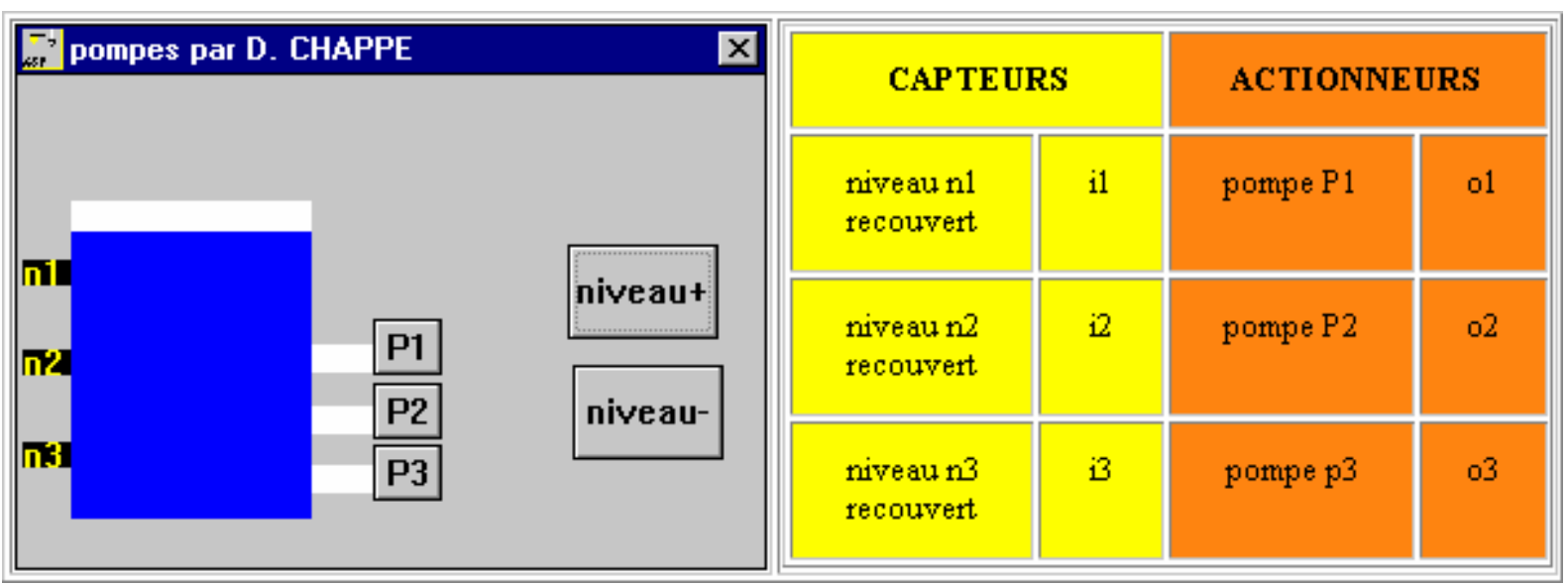

Figure 6. Modèle de simulation de la partie opérative.

\subsection{Traitement de surface (reprise de séquence)}

Une installation de traitement de surface comprend un chariot automoteur desservant quatre bacs, un poste de chargement et un poste de déchargement. Des capteurs _sq1_ à ${ }_{-} \mathrm{sq} 6$ _ permettent le positionnement au dessus des différents postes (Fig. 7).

Après avoir accroché les pièces à traiter sur le cadre situé au point de chargement en position basse, l'opérateur donne l'ordre de départ cycle. Le chariot doit alors effectuer le cycle décrit sur la figure 8. Vous pouvez voir la solution en annexe. 


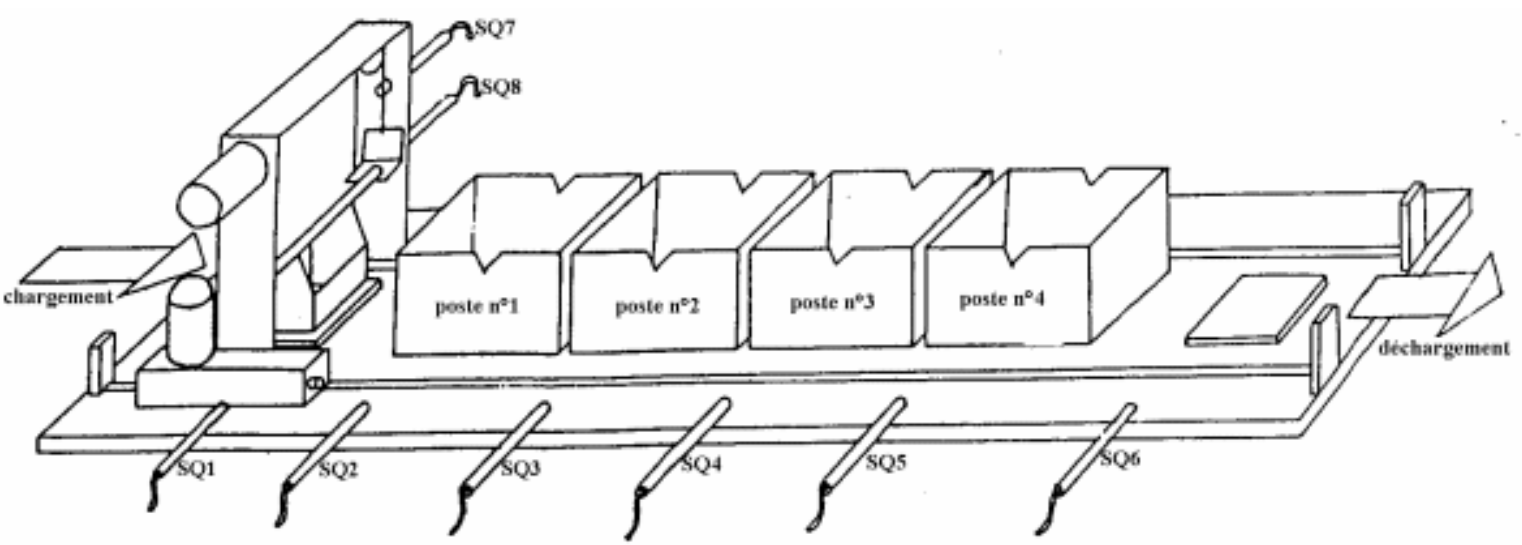

Figure 7. Ligne de traitement de surface.

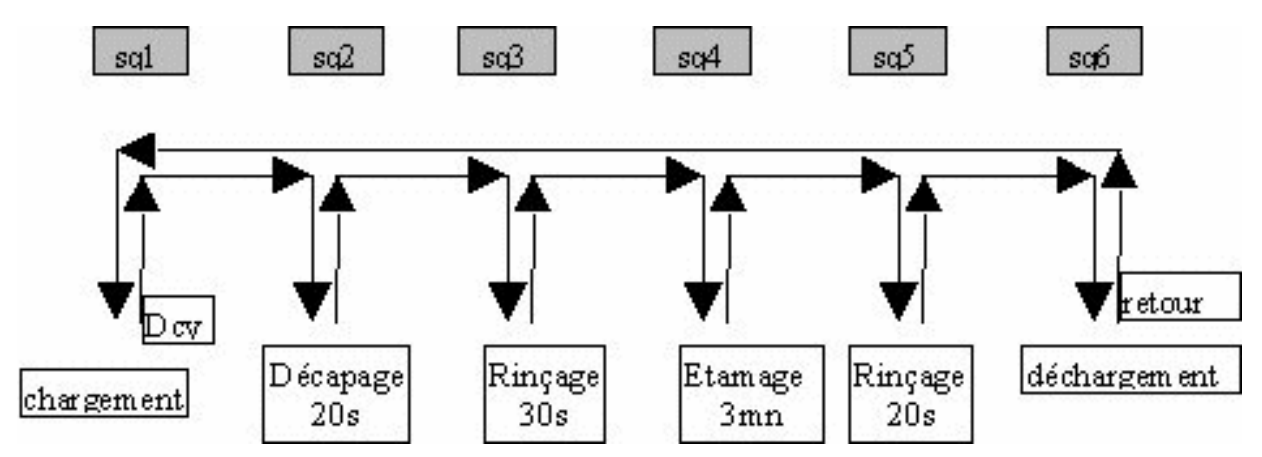

Figure 8. Cycle à réaliser.

\subsection{Presse à emboutir et son évacuation (synchronisation de séquences)}

Une presse à emboutir (Fig. 9) est alimentée par un dispositif formé par un tapis supérieur amenant les pièces dans un retourneur qui les retourne sur un tapis inférieur. Ce dernier élève les pièces jusqu'au niveau de la presse grâce à un ascenseur.

Après emboutissage une pince prend la pièce, préalablement surélevée par l'éjecteur, pour la déposer sur le tapis d'évacuation. La simulation utilise le modèle de la figure 10. Les capteurs et actionneurs utilisés sont donnés par le tableau 1. Vous pouvez voir une animation de la solution au format AVI (123 Ko) ou au format GIF animé $(48 \mathrm{Ko}) \mathbf{q}^{9}$. 


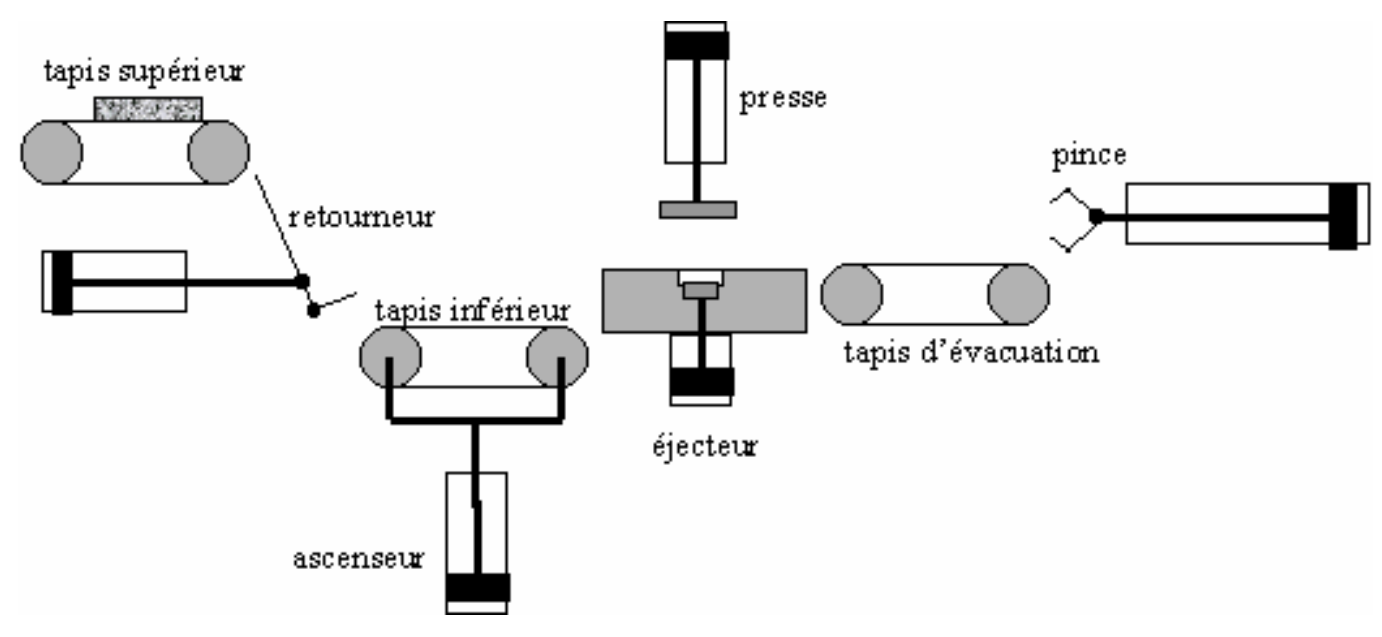

Figure 9. Presse et son alimentation.

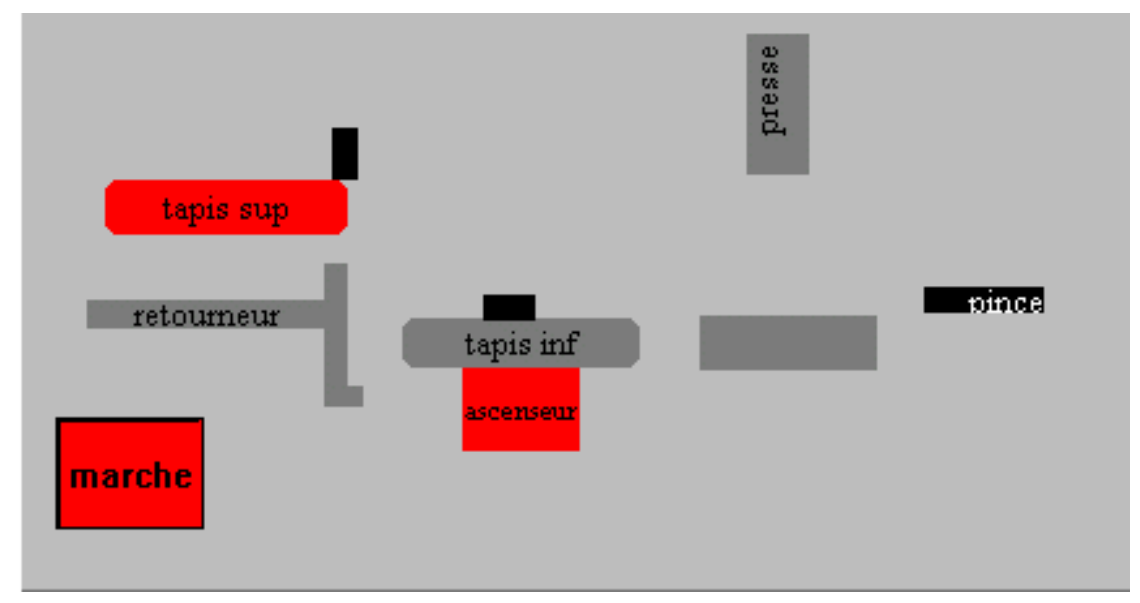

Figure 10. Modèle de simulation.

\begin{tabular}{|c|c|c|c|}
\hline \multicolumn{2}{|r|}{ Capteurs } & \multicolumn{2}{|r|}{ Actionneurs } \\
\hline dcy_ & marche & & \\
\hline ppr_ & pièce dans retourneur & RSUP_ & rotation tapis supérieur \\
\hline rre_ & retourneur reculé & RER_- & recul retourneur \\
\hline rav_ & retourneur avancé & AVR_ & avance retourneur \\
\hline ppa_ & présence pièce sur ascenseur & & \\
\hline haut__ & ascenseur en haut & $\mathrm{MAS}_{-}$ & montée ascenseur \\
\hline bas_ & ascenseur en bas & DAS_ & descente ascenseur \\
\hline ppp_ & présence pièce sous presse & RINF_ & rotation tapis inférieur \\
\hline pbas_ & presse en bas & _DPR_ & descente presse \\
\hline phaut & presse en haut & MPR_ & montée presse \\
\hline pav $_{-}$ & pince avancée & _APINCE & avance pince \\
\hline pre_ & pince reculée & _RPINCE_ & recul pince \\
\hline
\end{tabular}

Tableau 1. Capteurs et actionneurs de la presse à emboutir et de son évacuation. 


\section{Exemples génériques}

Nous présentons ici des exemples de solutions pour quelques problèmes types (gestion de priorités, partage de ressources, etc.). Ces solutions peuvent être réutilisées pour tout problème du même type.

\subsection{Gestion des priorités}

Une navette transporte un par un les produits fabriqués par trois lignes de fabrication indépendantes vers un tapis roulant d'évacuation selon le schéma de la figure 11. Les capteurs et actionneurs utilisés sont donnés par le tableau 2.

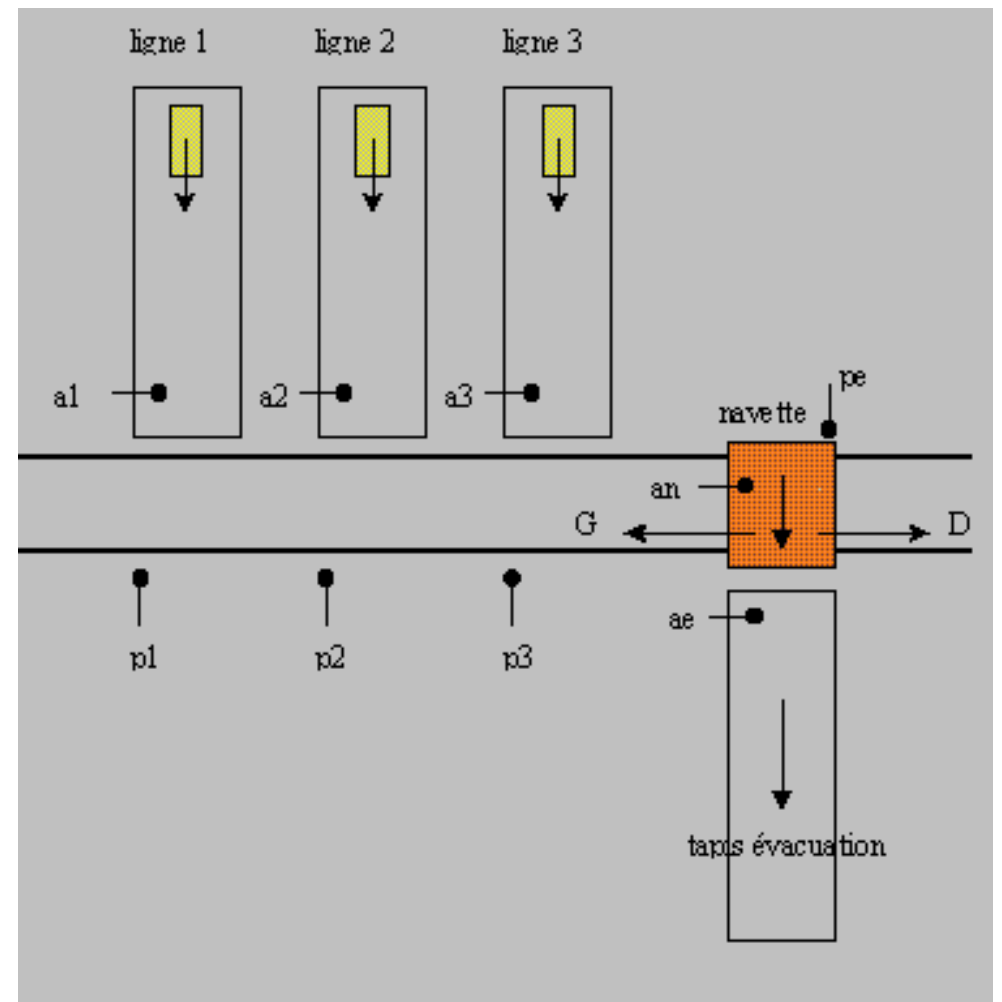

Figure 11.

\begin{tabular}{|l|l|l|l|}
\hline \multicolumn{2}{|c|}{ Capteurs } & \multicolumn{2}{c|}{ Actionneurs } \\
\hline a1 & présence pièce sur ligne 1 & G & déplacement de la navette vers la gauche \\
\hline a2 & présence pièce sur ligne 2 & D & déplacement de la navette vers la droite \\
\hline a3 & présence pièce sur ligne 3 & R1 & rotation tapis ligne 1 \\
\hline an & présence pièce sur navette & R2 & rotation tapis ligne 2 \\
\hline ae & présence pièce sur tapis évacuation & R3 & rotation tapis ligne 3 \\
\hline p1, p2, p3, pe & détecteurs positions de la navette & Rn & rotation tapis navette \\
\hline
\end{tabular}

Tableau 2. Capteurs et actionneurs de la gestions des priorités.

En donnant priorité à la ligne la plus éloignée on obtient le GRAFCET de la figure 12 dans lequel :

$$
\mathrm{u} 1=\mathrm{a} 1 \quad \mathrm{u} 2=/ \mathrm{a} 1 . \mathrm{a} 2 \quad \mathrm{u} 3=/ \mathrm{a} 1 . / \mathrm{a} 2 . \mathrm{a} 3 .
$$

Pour ne pas donner priorité toujours à la même ligne en cas de conflit on peut faire tourner les priorités en ajoutant un GRAFCET des priorités (Fig. 13). 


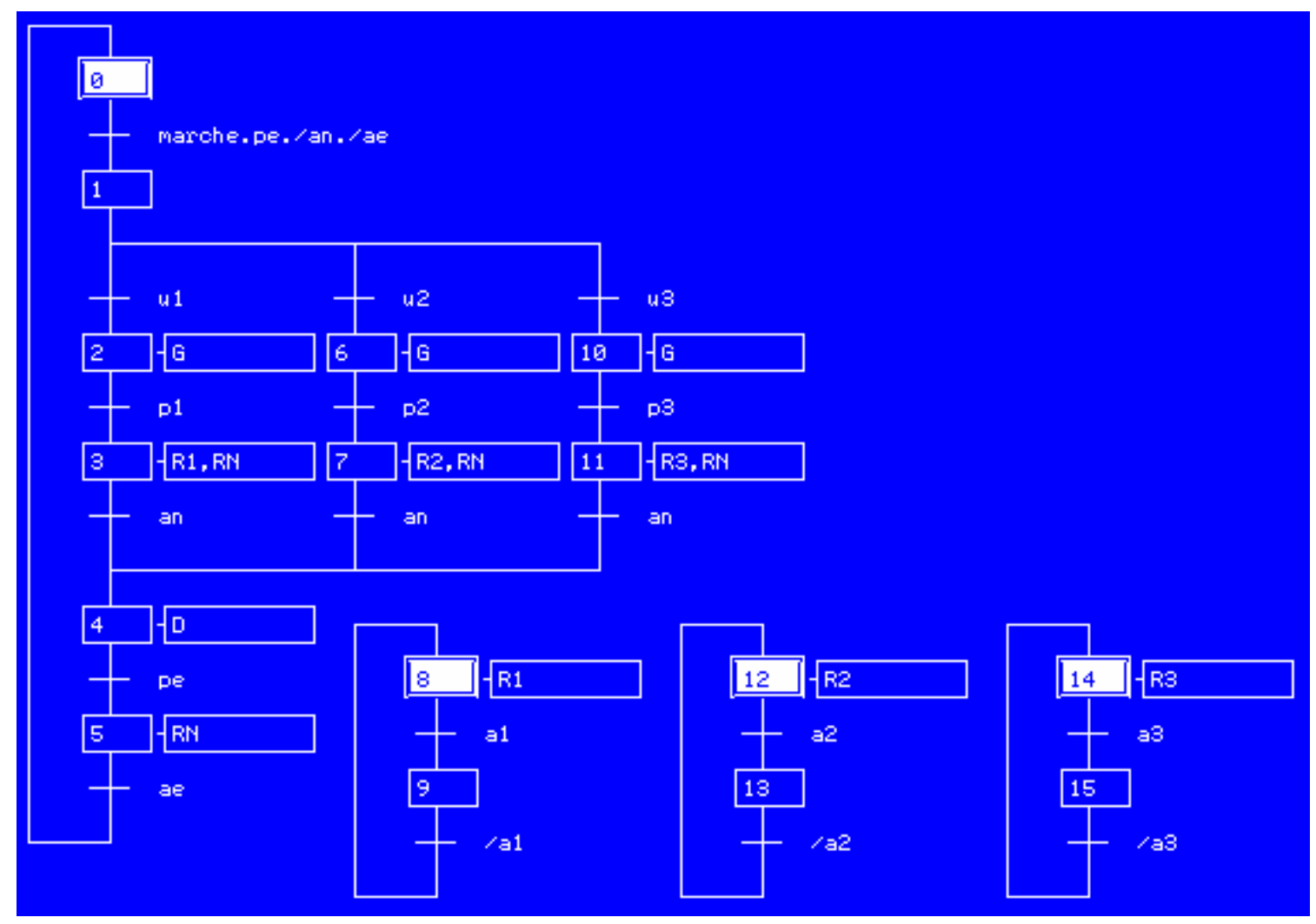

Figure 12. GRAFCET.

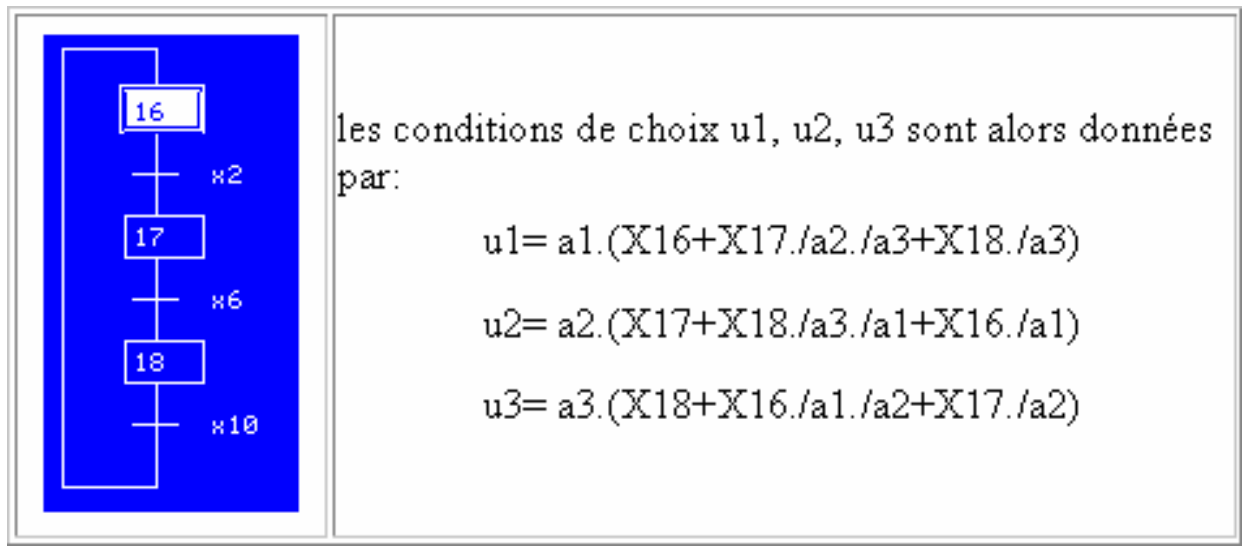

Figure 13. Gestion des priorités.

\subsection{Travail à la chaîne (d'après $P$. Trau [7])}

Soit une chaîne de remplissage de bidons d'huile (Fig. 14). Un tapis roulant se déplaçant par saccades, s'arrêtant à chaque nouvel appui de la came sur le capteur finav est alimenté manuellement sur le poste marqué entrée (de temps en temps il manque des bidons). Trois postes sont prévus : remplissage (R), bouchage (B) et enfoncement (E).

Un seul capteur (entrée) détecte la présence d'un bidon en début de chaîne. On désire faire les trois opérations simultanément, sauf s'il n'y a pas de bidon sous le poste. Il n'est pas nécessaire de rajouter des capteurs. On 
suppose que le tapis est vide lors de l'initialisation. La figure 15 donne les GRAFCET de pilotage de la chaîne. Vous pouvez voir une animation de la solution au format AVI (96 Ko) ou au format GIF animé (41 Ko) 9 ?.4.

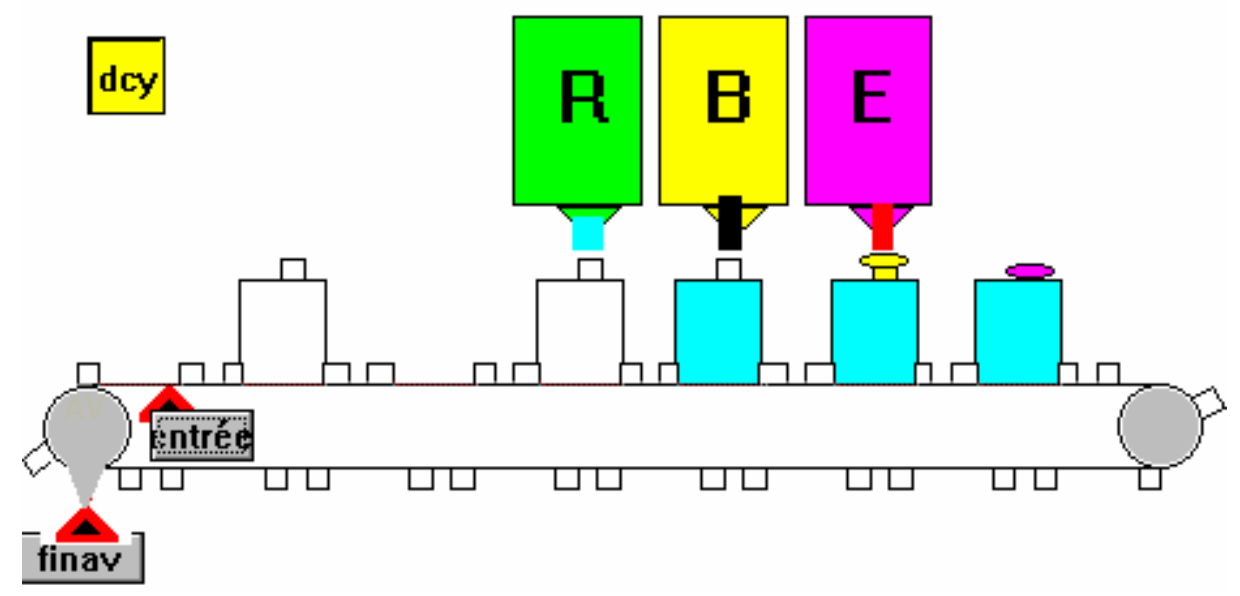

Figure 14. Chaîne de remplissage.

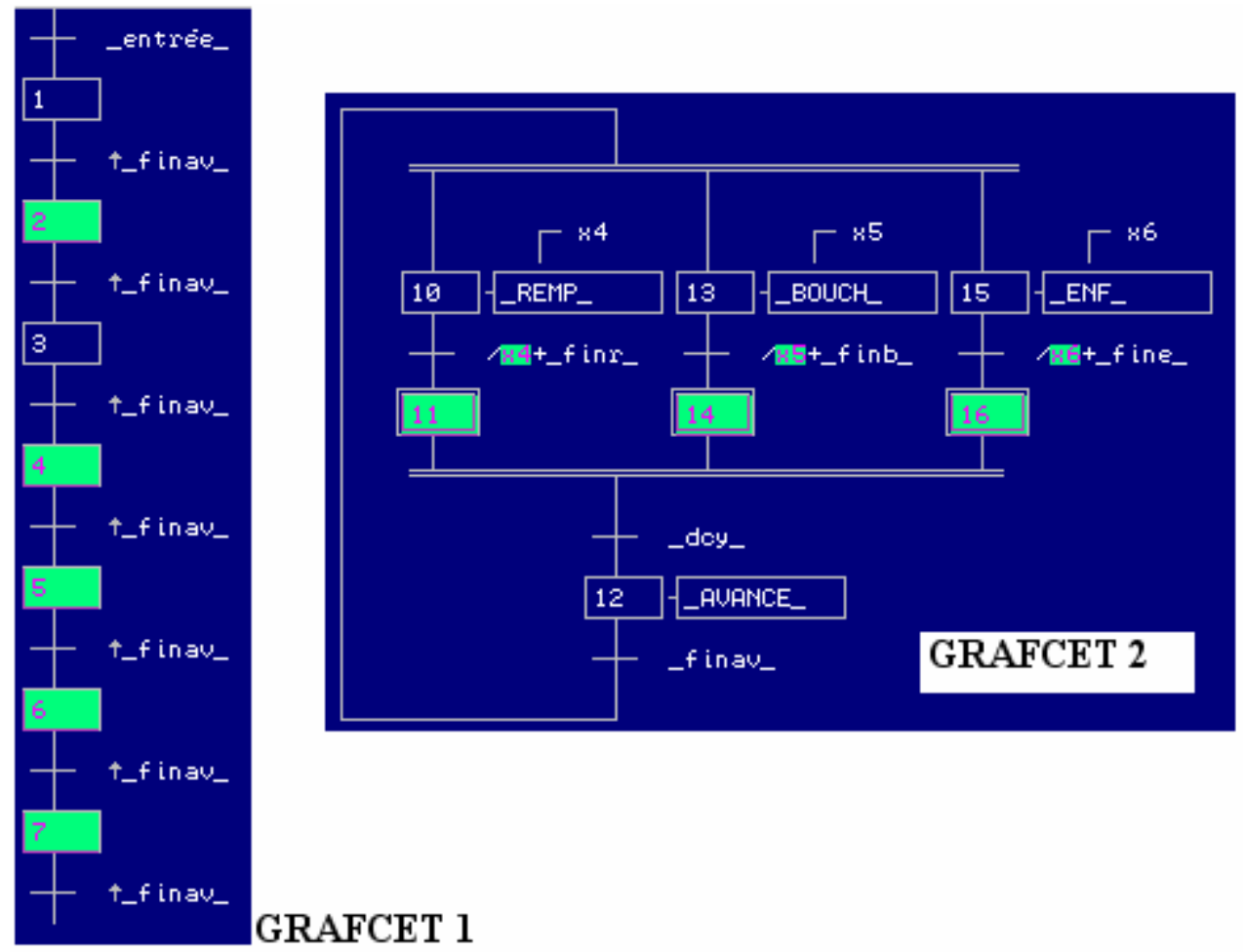

Figure 15. GRAFCET de pilotage de la chaîne.

(8 Ko-GIF)

Le GRAFCET 1 décrit l'évolution des bidons sur la chaîne : lorsqu'une étape $i$ est active, il y a un bidon au poste $i$. En fonctionnement normal toutes les étapes sont actives. La première transition est une transition source correspondant à l'entrée d'un bidon sur la chaîne. La dernière transition est une transition puits correspondant à la sortie d'un bidon de la chaîne.

Le GRAFCET 2 décrit les commandes des postes 4, 5, 6 et la commande de l'avance de la chaîne. 
Cette méthode utilise au mieux le séquencement du GRAFCET, on peut maintenant rajouter des capteurs, mais qui n'auront pour fonction que de vérifier le bon fonctionnement du système. Dans tous les cas similaires, on utilisera cette démarche.

\subsection{Partage de ressource (ou sémaphore)}

Deux wagonnets alimentent le skip de déchargement d'un haut-fourneau en empruntant une voie commune (Fig. 16).

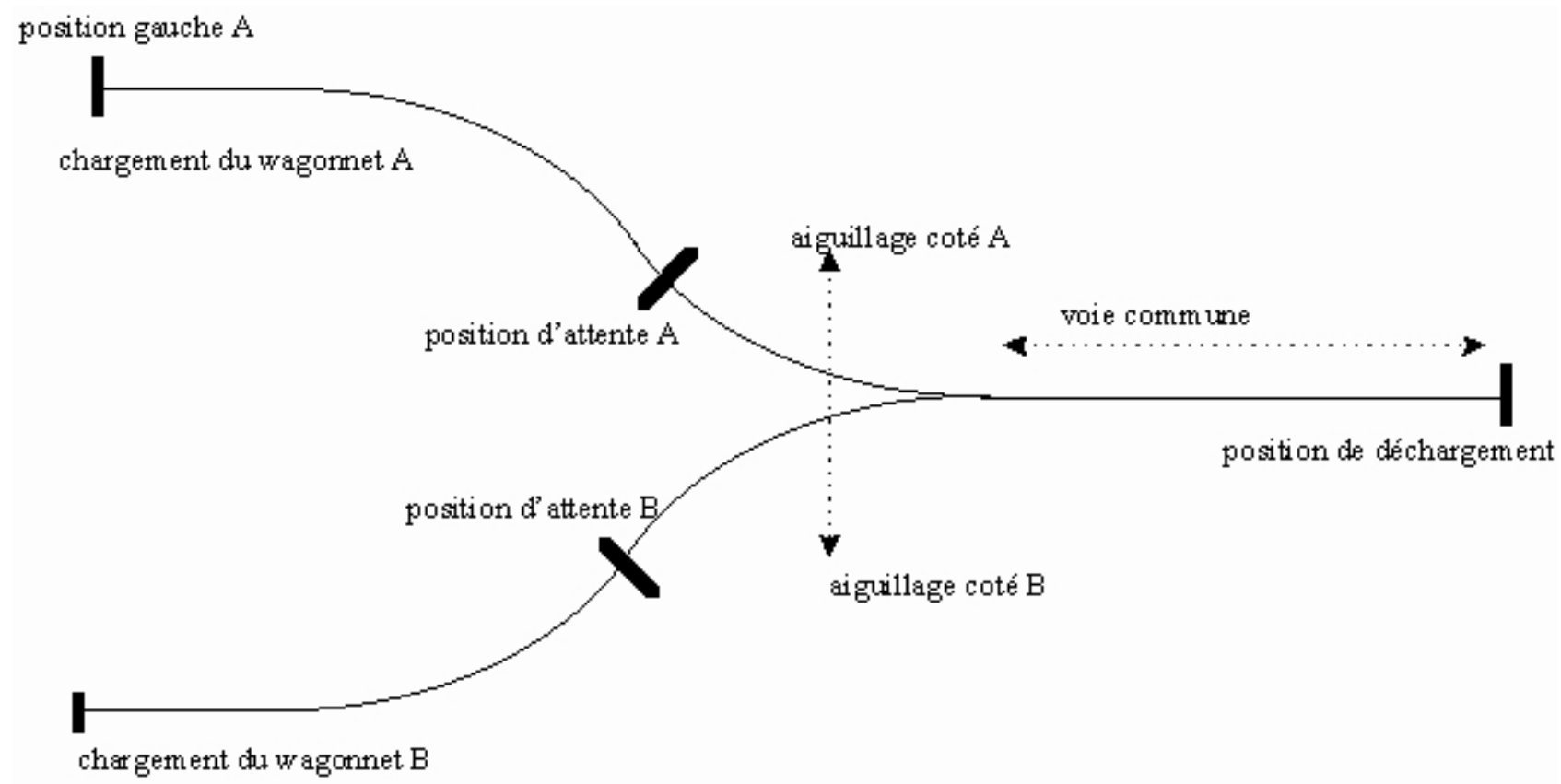

Figure 16. Partage de ressource.

Le cycle correspondant à un chariot est le suivant :

- dès que l'opérateur donne l'ordre de « départ cycle » (Dcy), le wagonnet considéré effectue automatiquement son chargement et démarre au signal fin de chargement $(\mathrm{FC})$.

- Le wagonnet se dirige ensuite vers la partie commune où il s'arrête à une position d'attente si la voie commune est occupée, sinon il faut positionner l'aiguillage sur la position correcte et le chariot continue.

- Arrivé à la position de déchargement automatique, il attend le temps nécessaire avant de retourner à la position initiale (attente $10 \mathrm{~s}$ ).

- Chaque déchargement de wagonnet est comptabilisé en vue d'une gestion journalière. 
Les capteurs et actionneurs utilisés sont donnés par le tableau 3.

\begin{tabular}{|c|c|c|c|}
\hline \multicolumn{2}{|r|}{ Capteurs } & \multicolumn{2}{|r|}{ Actionneurs } \\
\hline deya_ & départ wagonnet $\mathrm{A}$ & ava_ & avance wagonnet $\mathrm{A}$ \\
\hline dcyb_ & départ wagonnet B & avb_ & avance wagonnet B \\
\hline paa_ & position d'attente A & _aga_ & commande aiguillage sur pos A \\
\hline pab_ & position d'attente B & agb_ & commande aiguillage sur pos B \\
\hline paga & aiguillage en $\mathrm{A}$ & ara & retour wagonnet $\mathrm{A}$ \\
\hline pagb_ & aiguillage en B & arb_ & retour wagonnet B \\
\hline pdc_ & wagonnet en $\mathrm{C}$ & & \\
\hline fdch & fin de déchargement & & \\
\hline pca & wagonnet A en position de chargement & & \\
\hline pcb_ & wagonnet B en position de chargement & & \\
\hline
\end{tabular}

Tableau 3. Capteurs et actionneurs du partage de ressource.

La figure 17 donne une solution comportant trois GRAFCET (commande voie A, commande voie B, gestion de la ressource). La voie commune est une ressource physique que les deux utilisateurs ne peuvent pas utiliser en même temps. Dans les problèmes de ce type la disponibilité de la ressource commune ne peut pas être détectée par un capteur, mais uniquement par l'état du GRAFCET. Vous pouvez voir une animation de la solution au format AVI (105 Ko)

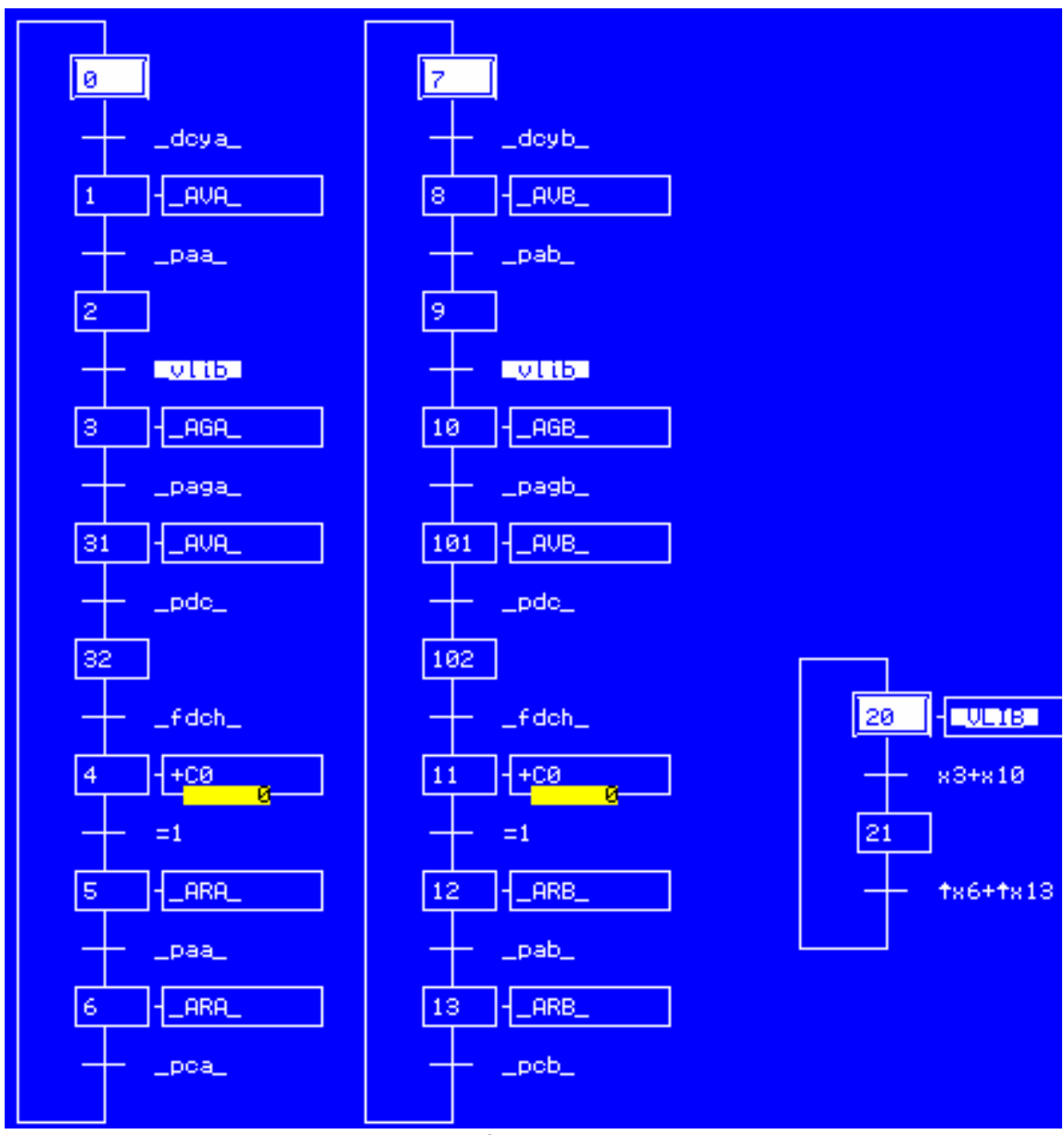

Figure 17. 


\section{Exemples ludiques}

\subsection{Parcours de labyrinthe}

Un objet mobile peut se déplacer selon quatre directions (haut, bas, droite, gauche) dans un labyrinthe et doit sortir automatiquement de ce labyrinthe. Il est muni de quatre capteurs ( sup, inf, c g, c d) activés lorsque le mobile entre en contact avec la paroi correspondante. Ce dispositif est commandé par un GRAFCET. Vous pouvez voir une animation de la solution au format AVI (74 Ko) ou au format GIF animé (24 Ko)

\subsection{Suivi d'une ligne}

Un objet mobile peut se déplacer selon 4 directions (haut, bas, droite, gauche). Il doit suivre une ligne continue constituée de segments horizontaux et verticaux.

Il est muni d'un seul capteur (i 0) activé lorsque le mobile est en contact avec la ligne.

Ce dispositif est commandé par un GRAFCET. Vous pouvez voir une animation de la solution au format AVI (59 Ko) ou au format GIF animé $(25 \mathrm{Ko})$

\section{Conclusion}

J'ai voulu faire ici un petit recueil d'exemples de commande par GRAFCET. La plupart de ces exemples sont classiques et ont été publiés dans des livres d'exercices ; mais je pense que l'animation des solutions, avec partie opérative simulée, peut apporter beaucoup à la compréhension du fonctionnement. J'espère que ces animations seront utilisées par les collègues qui enseignent le GRAFCET, et mises à la disposition de leurs étudiants. Les animations proposées ici pourraient sans doute être améliorées. Mais pour que les fichiers ne soient pas trop volumineux, j'ai dû limiter le nombre d'images par animation (on ne voit donc pas tous les pas successifs d'évolution du GRAFCET). La réalisation de chaque animation prend un temps non négligeable et il serait bon de se partager le travail et que d'autres apportent leurs idées et leur savoir-faire. C'est pourquoi j'invite tous les collègues qui ont fait une démarche du même type (ou qui souhaitent le faire) à mettre à la disposition de la communauté leurs travaux, par exemple par l'intermédiaire de cette revue.

\section{Références bibliographiques}

[1] Daniel Chappe, cours d'automatique,1998 [en ligne] http://www.lab.ens2m.fr/cours_automatique/index.html.

[2] Manuel de l'utilisateur AUTTOMGEN V.6 (IRAI, 1998).

[3] Manuel de l'utilisateur IRIS V.3 (IRAI, 1998).

[4] IRAI [en ligne] http://www.irai.fr. Adresse postale : IRAI, B.P. 14, 30110 La Grand' Combe.

[5] J. C. Bossy, P. Brard, P. Faugère et C. Merlaud, Le Grafcet, sa pratique et ses applications (Educalivre, Paris, 1979).

[6] M. Blanchard, Comprendre, maîtriser et appliquer le GRAFCET (CEPADUES Editions, 1979).

[7] P. Trau, cours d'automatique [en ligne]

http://www-ipst.u-strasbg.fr/pat/autom/grafcet.htm.

Les liens Internet sont tous valides à la date du 26 novembre 2001. 
Annexe figure8

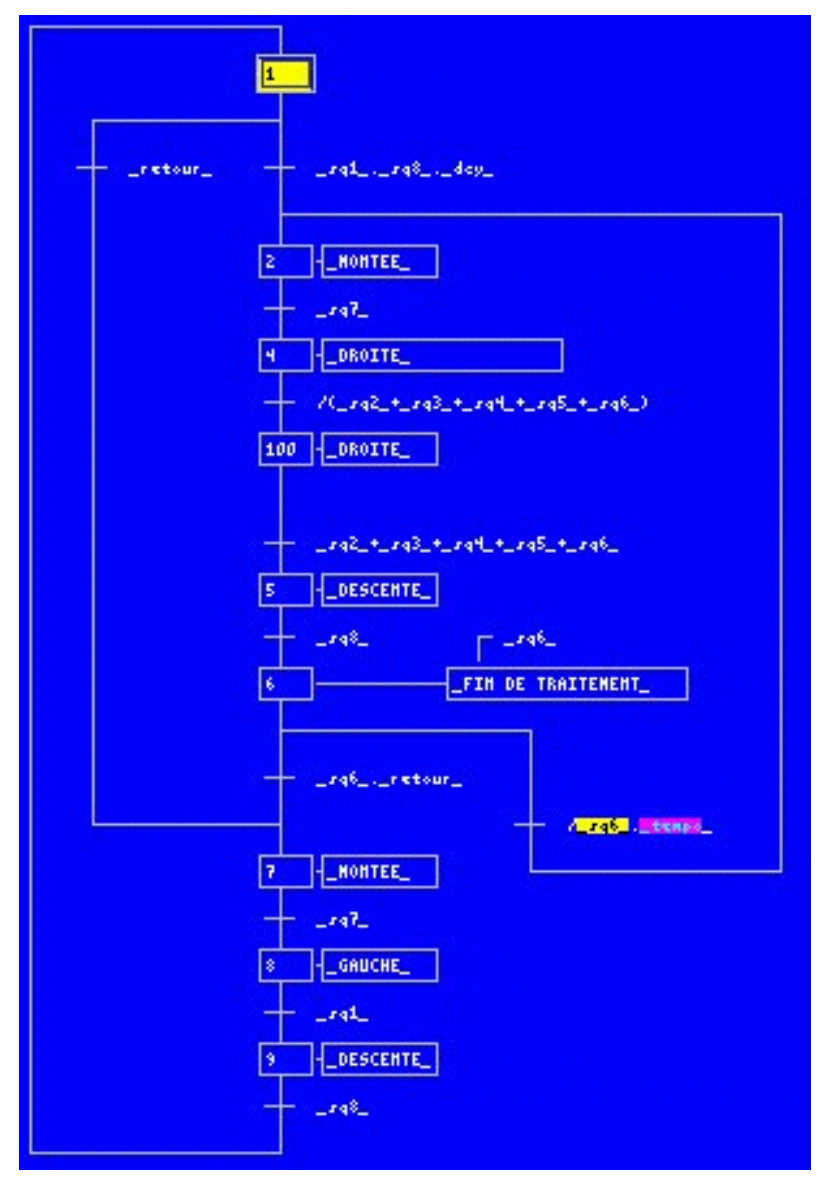

